\title{
Thermodynamic behavior of nanomagnets with a vortex configuration
}

\author{
R. Wieser and K. D. Usadel \\ Theoretische Physik, Universität Duisburg-Essen, 47048 Duisburg, Germany \\ U. Nowak \\ Department of Physics, University of York, York YO105DD, United Kingdom
}

\begin{abstract}
The thermodynamic behavior of flat circular nanomagnets with a vortex domain configuration is studied using Langevin dynamics simulations for the dynamical behavior as well as local mean-field calculations for equilibrium properties. Our studies show that the vortex core becomes thermally unstable with increasing temperature, acting like a superparamagnetic system. On time scales where the vortex core remains within one of the metastable states it still has a stronger temperature dependence than the magnetization far away in the bulk of a domain.
\end{abstract}

PACS number(s): 75.10.Hk, 75.40.Mg, 75.60.Ch

\section{INTRODUCTION}

Nanostructured ferromagnetic elements are important constituents for future data storage devices, biosensors, or other spintronic devices. ${ }^{1}$ For applications as memory elements its magnetic state must be well-defined and stable. With decreasing size of the magnetic structures, however, the stability of the magnetic state with respect to thermal fluctuations decreases and, consequently, any stored information gets lost. Different possibilities are discussed in the literature to enhance the thermal stability or, alternatively, to increase the amount of information which is stored in one nanoelement, for instance, by using disk shaped elements exhibiting a vortex domain state. Indeed, it has been argued that the independence of the central polarization on the vorticity makes it possible to use such vortex structures for storing two bits instead of only one $\mathrm{e}^{2}$ in a single nanoelement.

The increasing interest in vortex domain structures has lead to recent investigations of flat cylindrical nanomagnets ${ }^{3-8}$ where the theoretical work ${ }^{5-7}$ has been done within the framework of a micromagnetic approach, neglecting the influence of finite temperatures. However, reducing the size of the magnetic structures thermodynamic fluctuations are getting increasingly important. Therefore in the present work we focus on the thermodynamical stability of vortex domain structures using a local spin model. This approach enables us to describe the magnetic structure of a vortex including thermal fluctuations and overcoming any discretization problems. ${ }^{6}$ We investigate the magnetic stability of a vortex core using Langevin dynamic simulations for the dynamic behavior as well as local mean field calculations for the equilibrium thermodynamics.

\section{MODEL}

Our aim is to model thermal behavior of a ferromagnet for temperatures up to the Curie temperature. In order to properly simulate these elevated temperatures where short wavelength spin waves are relevant one needs an atomic resolution. Therefore we consider a classical spin model on an atomistic level which takes care of spin waves of all possible wavelengths. We take into account energy contributions from nearest-neighbor exchange and dipole-dipole interaction with a Hamiltonian

$$
\mathcal{H}=-\frac{J}{2} \sum_{i, j} \mathbf{S}_{i} \cdot \mathbf{S}_{j}-\frac{\omega}{2} \sum_{i, j} \frac{3\left(\mathbf{S}_{i} \cdot \mathbf{e}_{i j}\right)\left(\mathbf{e}_{i j} \cdot \mathbf{S}_{j}\right)-\mathbf{S}_{i} \cdot \mathbf{S}_{j}}{r_{i j}^{3}}
$$

Here, the $\mathbf{S}_{i}=\mu_{i} / \mu_{s}$ are three-dimensional unit vectors located on a simple-cubic lattice. $J$ denotes the nearestneighbor exchange coupling constant while $\omega$ $=\mu_{0} \mu_{s}^{2} /\left(4 \pi a^{3}\right)$ is the strength of the dipolar interaction with the atomic magnetic moment $\mu_{s}$ and the lattice constant $a$. We neglect any contributions from crystalline anisotropies restricting ourselves to the minimal model which can display a vortex domain structure as ground state. Furthermore, our investigation is in zero external magnetic field.

The systems to be studied are flat cylinders with a height of 4 lattice constants and diameters of 35 and 32 lattice constants, respectively. In the first case the center of the cylinder coincides with the central lattice sites while in the second case it is in between the group of central lattice sites. For this geometry and those values for the parameter $\omega$ used here the ground state is found to be a vortex state with a central portion having a magnetization with a large component perpendicular to the cylinder plane. This magnetization can point into two directions resulting in a ground state which is fourfold degenerated. Note that for an uneven number of spins the four spins (one per plane) in the center of the cylinder are strictly perpendicular to the cylinder plane while in the other case the central spins are slightly twisted with respect to each other.

The dynamics of the spin system at finite temperatures is governed by the stochastic Landau-Lifshitz-Gilbert (LLG) equation,

$$
\frac{\partial \mathbf{S}_{i}}{\partial t}=-\frac{\gamma}{\left(1+\alpha^{2}\right) \mu_{s}} \mathbf{S}_{i} \times\left[\mathbf{H}_{i}(t)+\alpha\left(\mathbf{S}_{i} \times \mathbf{H}_{i}(t)\right)\right],
$$

with the gyromagnetic ratio $\gamma$, the dimensionless damping constant $\alpha$ (after Gilbert), and the internal fields $\mathbf{H}_{i}(t)$ $=-\partial \mathcal{H} / \partial \mathbf{S}_{i}+\zeta_{i}(t)$, where $\zeta_{i}(t)$ is a thermal noise term. The noise $\zeta_{i}(t)$ represents thermal fluctuations, with $\left\langle\boldsymbol{\zeta}_{i}(t)\right\rangle=0$ and 


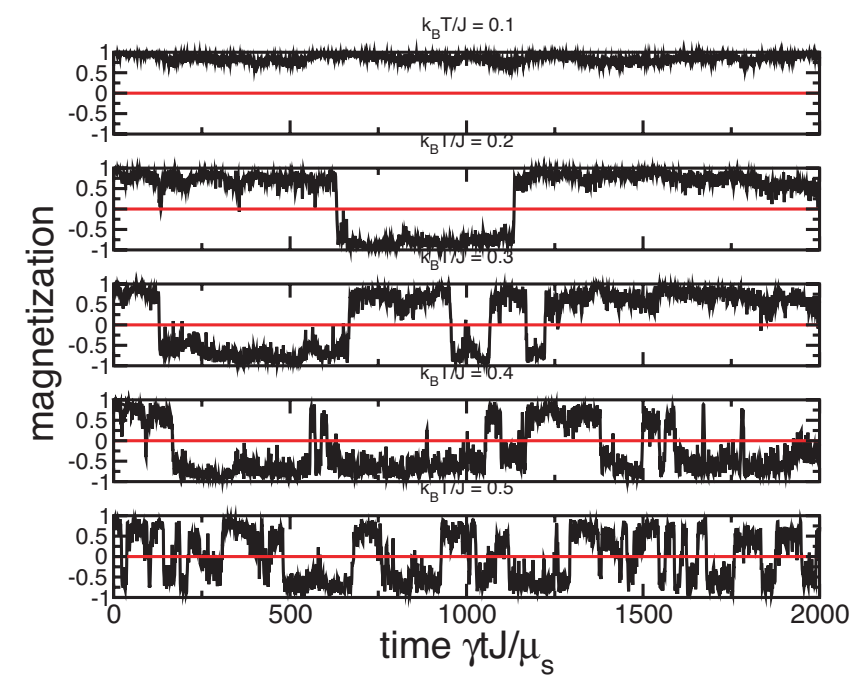

FIG. 1. (Color online) Superparamagnetic behavior of the vortex core: Magnetization of the vortex core vs time for different temperatures. The system diameter is 35 spins, $\omega / J=0.2$.

$\left\langle\zeta_{i}^{\nu}(t) \zeta_{j}^{\theta}\left(t^{\prime}\right)\right\rangle=2 \delta_{i j} \delta_{\nu \theta} \delta\left(t-t^{\prime}\right) \alpha k_{B} T \mu_{s} / \gamma$ where $i, j$ denotes lattice sites and $\nu, \theta$ Cartesian components. In order to compute the long-range dipole-dipole interaction efficiently we use fast Fourier transformation methods. All methods involved are described in detail in. Ref. 9

The larger the ratio of dipolar vs exchange interaction, $\omega / J$, the smaller is the size of the vortex core. In the following we use values of $\omega / J$ ranging from 0.04 to 0.3 leading to sufficiently small vortex cores which fit into system sizes which can be simulated with an atomic resolution so that the thermodynamics of the vortex can be investigated. In this limit the size of the vortex core depends on the thickness (which we do not vary in the following) and on the value of $\omega / J$ rather than on the radius of the cylinder which is clearly larger the the size of the vortex core. We would like to point out that such high values for $\omega / J$ which we investigate here can only occur in rare earth metals while in common ferromagnetic materials $\omega / J$ is much smaller. However, our study is intended to have a general character rather than being specific for any type of experimental material.

\section{SUPERPARAMAGNETIC BEHAVIOR}

First, the finite temperature dynamic behavior of these systems is studied numerically using Langevin dynamics simulations, ${ }^{9}$ i.e., numerical solutions of Eq. (2). Special emphasis is laid on the behavior of the vortex core. Figure 1 shows the time evolution of the magnetization of the vortex core, averaged over the central spins and normalized to unity. The simulations start from one of the ground states with the core magnetization pointing upwards. For lower temperatures the magnetization of the core is stable over a long time period before an abrupt switching event to the opposite direction occurs. For the case of single-domain nanoparticles this kind of behavior is known as superparamagnetism. Here, analogously, the two energetically equal states of the vortex core are separated by a finite energy barrier which can be

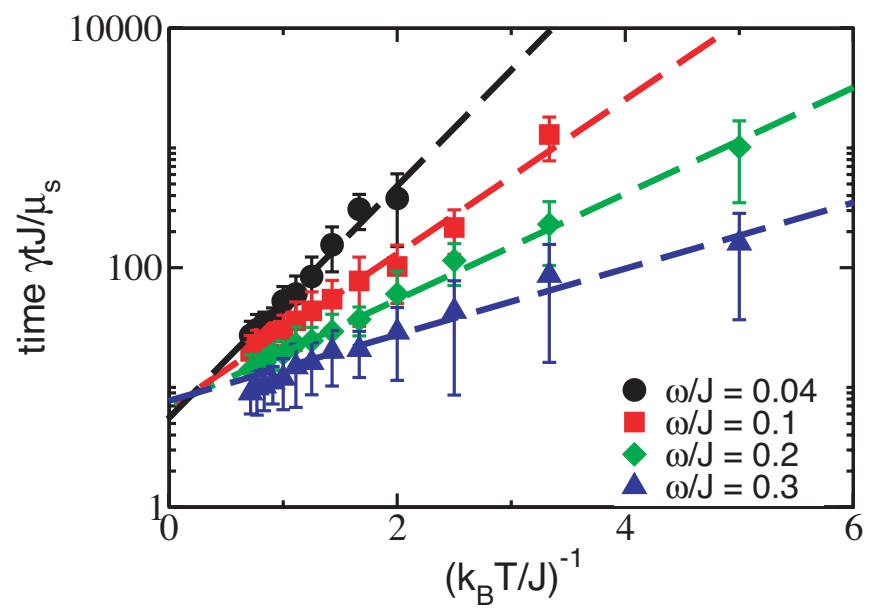

FIG. 2. (Color online) First switching time vs inverse temperature for different values of $\omega / J$. The system diameter is 35 spins.

overcome by thermal fluctuations. The switching time decreases with increasing temperatures and at the same time the fluctuations of the magnetization increase.

To describe this effect in more detail we investigate the mean first switching time, defined as the time between starting the simulation with up magnetization and switching to down magnetization averaged over 10000 runs. Figure 2 shows this time as a function of inverse temperature up to the Curie temperature. The low temperature data can be reasonably fitted by a straight line showing that the switching time can be described rather well by a thermal activation law,

$$
\tau=\tau_{0} e^{\Delta E_{b} / k_{B} T}
$$

with an energy barrier $\Delta E_{b}$. Note that such a simple law is expected to be valid for temperatures much lower than the energy barrier-a condition which is hard to fulfill in a computer simulation. Consequently, we cannot rule out that an analysis for lower temperatures would change the numerical values for the energy barriers which we obtain, or even the reversal mode (see, e.g., Ref. 10 for an investigation of energy barriers in a system with several different reversal modes).

However, the following table contains the values of $\Delta E_{b}$ following from the fitting procedure above:

\begin{tabular}{cllll}
$\omega / J$ & 0.04 & 0.1 & 0.2 & 0.3 \\
\hline$\Delta E_{b} / J$ & 2.27 & 1.486 & 1.018 & 0.565 \\
Error bar & 0.13 & 0.058 & 0.023 & 0.031
\end{tabular}

Obviously, the energy barrier decreases with increasing ratio of $\omega / J$. In other words it increases with the size of the vortex core.

In the low temperature limit the energy barrier is given by the energy difference between a vortex configuration and that transient state with the highest energy during the reversal. The energy barrier and in this way the stability increases with increasing core radius. This fact can be used to control the stability of the vortex by controlling the size of the core 
radius which can be achieved via the geometry (thickness and radius) of the cylinder ${ }^{2}$ or the $\omega / J$ ratio.

The obvious interpretation of these results is that the energy of the system has local minima in which the system stays for long times at sufficiently low temperatures. However, since the system considered is finite switching to the other local minima must occur as the result of thermal fluctuations for any finite temperature. In the following we will in detail investigate the local free energy minima for finite temperatures.

\section{METASTABLE STATES}

It has been shown by Garanin ${ }^{11,12}$ that the thermally averaged magnetization fulfills an equation similar to the LLG equation [Eq. (2)] but without the noise term. The corresponding equation of motion is called Landau-Lifshitz-Bloch (LLB) equation. This equation contains both the transverse as well as the longitudinal relaxation of the magnetization. In other words, in contrast to the LLG equation the magnetization vector is not assumed to be of constant length (in space and time). Invoking then a mean-field type approximation for the interacting spins the stationary points of the LLB equation are the local minima of the mean-field free energy. Hence the LLB equation links mean-field thermodynamic equilibrium properties with the dynamics of magnetic systems.

From a study of a mean-field temperature dependent behavior of magnetic domain structures interesting information about the stability of these structures can be deduced. Therefore in the following we study the mean-field behavior of vortex domain structures using the following local meanfield Hamiltonian (omitting terms without $\mathbf{S}_{i}$ ),

$$
\mathcal{H}_{\mathrm{MF}}^{-}=-\frac{J}{2} \sum_{i, j} \mathbf{S}_{i} \cdot \mathbf{m}_{j}-\frac{\omega}{2} \sum_{i} \mathbf{S}_{i} \cdot \mathbf{H}_{i}^{\text {dipole }},
$$

which corresponds to Eq. (1). Here, $\mathbf{m}_{i}$ is the local mean field at the lattice site $i$. The dipolar field $\mathbf{H}_{i}^{\text {dipole }}$ is given by

$$
\mathbf{H}_{i}^{\text {dipole }}=\sum_{j \neq i} \frac{3\left(\mathbf{e}_{i j} \cdot \mathbf{m}_{j}\right) \mathbf{e}_{i j}-\mathbf{m}_{j}}{r_{i j}^{3}} .
$$

The local magnetization at lattice site $i$ can consequently be calculated iteratively by numerically solving the equation

$$
\mathbf{m}_{i}=\left\langle\mathbf{S}_{i}\right\rangle=\frac{\operatorname{Tr} \mathbf{S}_{i} e^{-\beta \mathcal{H}_{\mathrm{MF}}^{-}}}{\operatorname{Tr} e^{-\beta \mathcal{H}_{\mathrm{MF}}^{-}}} .
$$

Here, we use $\beta=1 / k_{\mathrm{B}} T$, the trace is an integral over the unit sphere and the dipolar field is once again calculated by fast Fourier transformation methods.

In the case of a local mean-field calculation the system stays in one of the metastable states and the dependence of the magnetization on temperature within this state can be observed. The results can be interpreted as a "short time magnetization," averaged on time scales between switching processes (see once again Fig. 1).

In Fig. 3 we compare the temperature dependence of different magnetization components within the vortex core and
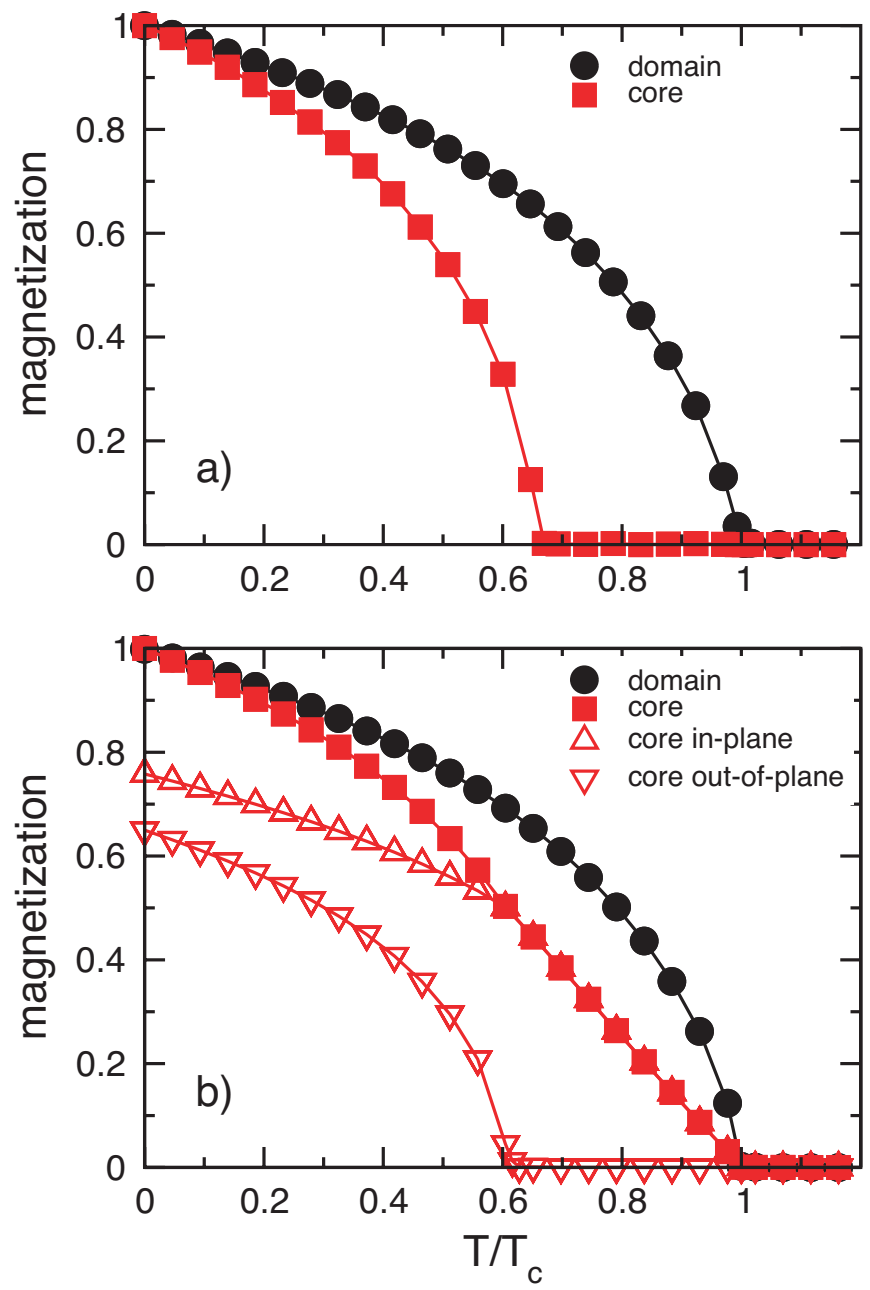

FIG. 3. (Color online) Temperature dependence of different magnetization components inside and outside the vortex core for the case of a system diameter of (a) 35 spins and (b) 32 spins. $\omega / J=0.2$.

in the bulk of the system. In the case of a system diameter of 35 spins we have one central spin (per plane), as before in the previous section, with a magnetization which is perpendicular to the cylinder plane. The core magnetization shown in Fig. 3(a) is the absolute value of its magnetization, averaged over the four planes. The domain magnetization is the magnetization of spins far away from both the core and the system boundary. This magnetization is mainly in-plane and resembles the usual bulk magnetization of an infinite system. Note that both magnetization values are different and that they have a different critical temperature.

In the case of a system diameter of 32 spins [Fig. 3(b)] we find four central spins (per plane) disposed around the geometrical center of the system. Since these spins are canted they consequently have both in-plane and out-of-plane magnetization components. Therefore in Fig. 3(b) we show, in addition to the absolute value of the core magnetization, its in-plane $\left(S_{i}^{\text {in }} \equiv \sqrt{\left(S_{i}^{\chi}\right)^{2}+\left(S_{i}^{y}\right)^{2}}\right)$ and out-of-plane component $\left(S_{i}^{\text {out }} \equiv S_{i}^{z}\right)$ as well.

In both cases we observe a much stronger decrease of the magnetization as a function of temperature in the core region 

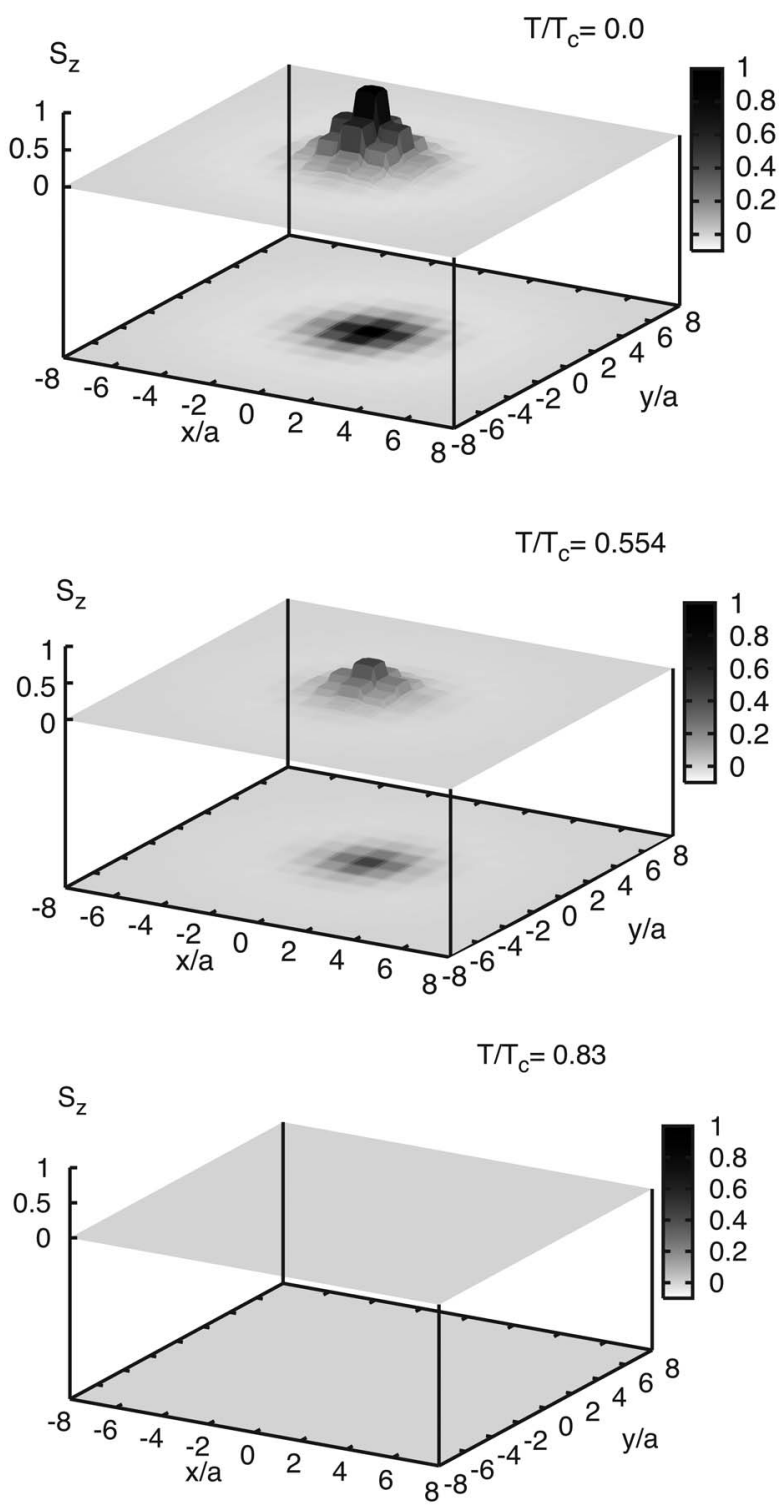

FIG. 4. Breakdown of the core magnetization: out-of-plane magnetization in the central part of the disk for different temperatures corresponding to Fig. 3(a).

as a compared to the region far away from the core. For temperatures larger than a characteristic temperature $T_{h}$ up to the higher Curie temperature $T_{c}$ we observe a vortex with zero out-of-plane magnetization. Note that in the region below $T_{h}$ only a small decrease of the in-plane magnetization not only outside the vortex but also in the center of the vortex is observed. With increasing temperatures the magnetization decreases further and it vanishes at $T_{c}$ both inside and outside the vortex core.
The breakdown of the out-of-plane magnetization in the vortex core is further illustrated in Fig. 4. Here, the spatially resolved magnetization in the central part of the disk is shown, averaged over all four planes. Note especially that there is no out-of-plane magnetization in the lowest graph which corresponds to a temperature above $T_{h}$ but still is well below $T_{c}$.

The mechanism behind this effect, the vanishing of a magnetization component within a domain wall below $T_{c}$, can be traced back to a lowering of the exchange interaction by changing the angle between the interacting spins. In the regime far away from the core the neighboring spins are almost parallel. In the vortex core the angle between the magnetic moments is large if they stay in plane and can be lowered by going out of plane thus lowering the exchange interaction. With increasing temperatures the magnetization decreases and the gain in exchange energy becomes more unimportant resulting in a transition to an in-plane configuration. A similar effect occurs in planar domain walls which undergo a transition from their zero-temperature behavior via elliptic walls to linear walls with increasing temperature. ${ }^{13-15}$

These mean-field results help also to understand the switching behavior of our disks further. For $T<T_{h}$ the system has two local minima of the free energy between which it switches by thermal activation. The corresponding energy barrier itself is a thermodynamic quantity. In Ref. 16 it was shown for the case of single-domain superparamagnetic FePt particles that the energy barrier vanishes thermodynamically at the Curie temperature. In our case it decreases with increasing temperature and it must vanish at $T_{h}$.

\section{SUMMARY}

To summarize, we have shown that with increasing temperature magnetic vortex structures in flat cylindrical nanomagnets become unstable. We have found two different effects acting on two different time scales and at different temperature regimes.

At a shorter time scale and in the temperature regime near the Curie temperature $T_{c}$ we have a breakdown of the outof-plane magnetization component of the core magnetization, an effect which corresponds to the thermodynamic transition to linear domain walls in one-dimensional magnetization profiles. ${ }^{13-15}$ At a longer time scale and at lower temperatures we demonstrated the possibility of a superparamagnetic behavior of the core of a vortex domain configuration.

\section{ACKNOWLEDGMENT}

This work was supported by the Deutsche Forschungsgemeinschaft through SFB 491. 
${ }^{1}$ R. P. Cowburn, D. K. Koltsov, A. O. Adeyeye, M. E. Welland, and D. M. Tricker, Phys. Rev. Lett. 83, 1042 (1999).

${ }^{2}$ A. Killinger, R. Höllinger, and U. Krey, J. Magn. Magn. Mater. 272-276, 724 (2004).

${ }^{3}$ T. Shinjo, T. Okuno, R. Hassdorf, K. Shigeto, and T. Ono, Science 289, 930 (2000).

${ }^{4}$ A. Wachowiak, J. Wiebe, M. Bode, O. Pietzsch, M. Morgenstern, and R. Wiesendanger, Science 298, 577 (2002).

${ }^{5}$ K. Y. Guslienko, B. A. Ivanov, V. Novosad, Y. Otani, H. Shima, and K. Fukamichi, J. Appl. Phys. 91, 8037 (2002).

${ }^{6}$ A. Thiaville, J. M. Garcia, R. Dittrich, J. Miltat, and T. Schrefl, Phys. Rev. B 67, 094410 (2003).

${ }^{7}$ R. Hertel and J. Kirschner, J. Magn. Magn. Mater. 270, 364 (2003).

${ }^{8}$ H. F. Ding, A. K. Schmid, D. Li, K. Y. Guslienko, and S. D.
Bader, Phys. Rev. Lett. 94, 157202 (2005).

${ }^{9}$ U. Nowak, in Annual Reviews of Computational Physics IX, edited by D. Stauffer (World Scientific, Singapore, 2001), p. 105.

${ }^{10}$ D. Hinzke and U. Nowak, Phys. Rev. B 61, 6734 (2000).

${ }^{11}$ D. A. Garanin, Physica A 172, 470 (1991).

${ }^{12}$ D. A. Garanin, Phys. Rev. B 55, 3050 (1997).

${ }^{13}$ L. N. Bulaevskiı̌ and V. L. Ginzburg, Sov. Phys. JETP 18, 530 (1964).

${ }^{14}$ J. Kötzler, D. A. Garanin, M. Hartl, and L. Jahn, Phys. Rev. Lett. 71, 177 (1993).

${ }^{15}$ N. Kazantseva, R. Wieser, and U. Nowak, Phys. Rev. Lett. 94, 037206 (2005).

${ }^{16}$ U. Nowak, O. N. Mryasov, R. Wieser, K. Guslienko, and R. W. Chantrell, Phys. Rev. B 72, 172410 (2005). 